ASME Turbo Expo 2017

\title{
Scale-resolving simulations of bypass transition in a high-pressure turbine cascade using a spectral-element discontinuous-Galerkin method
}

Anirban Garai, Laslo Diosady, Scott Murman, Nateri Madavan

NASA Ames Research Center
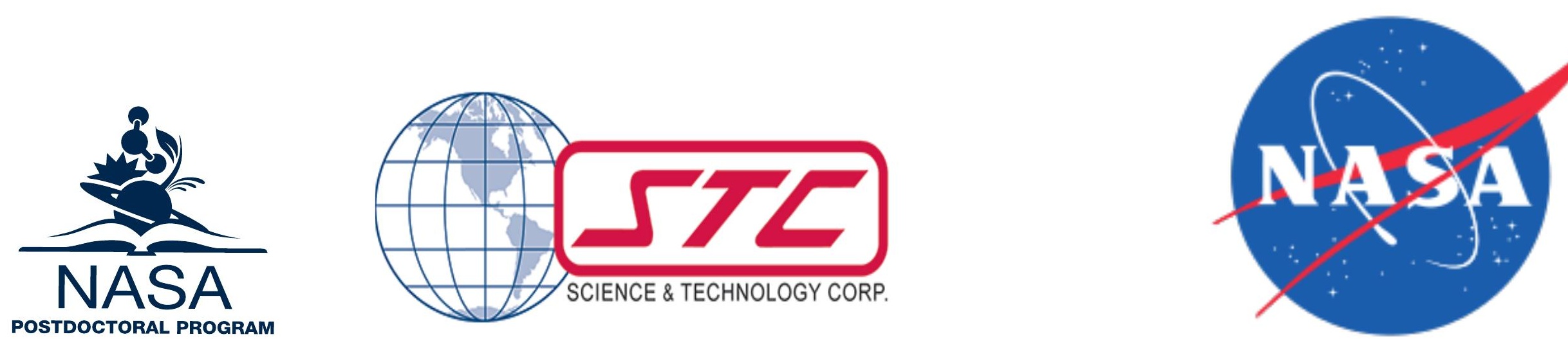


\section{Introduction}

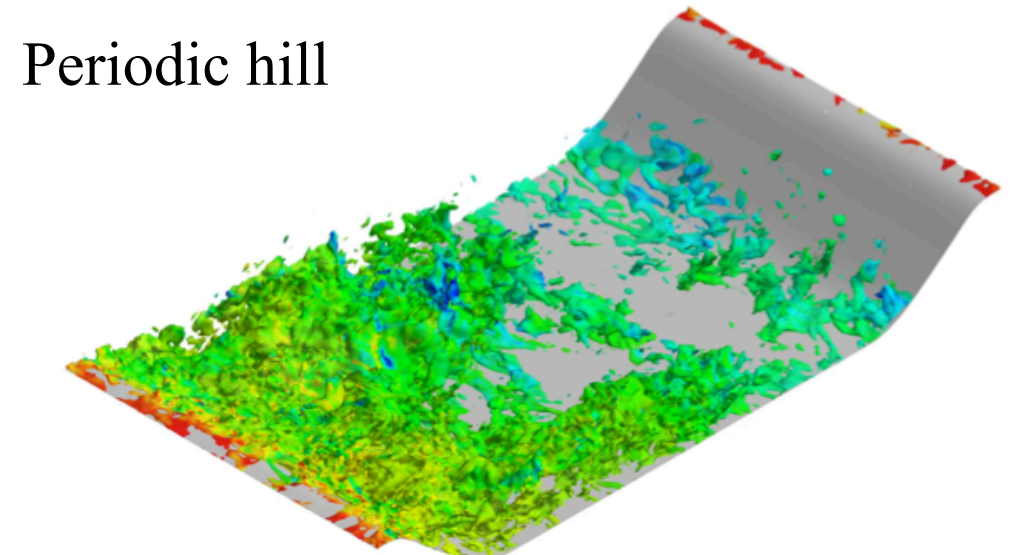

Diosady, Murman 2015, AIAA paper 2014-2784

LPT

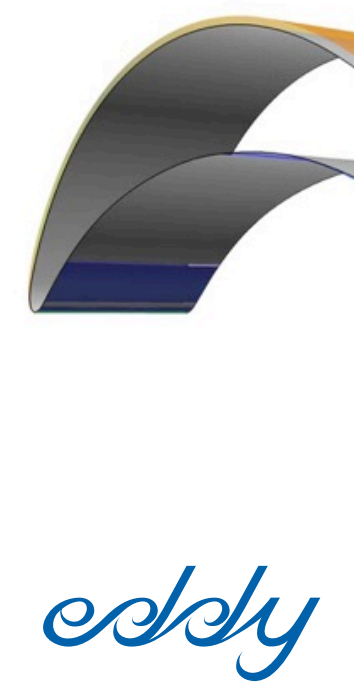

- Develop high-fidelity DNS/LES methods for next-generation computer architectures for turbomachinery analysis (and design)

- Higher-order Discontinuous Galerkin (DG) methods are the subject of considerable research

- Both space and time treated in a similar fashion

- Complex geometry handling

- Robust with respect to unstructured mesh quality

- Efficient implementation on modern exascale hardware

- Extends to arbitrary orders of accuracy in space and time

- Code has been used to simulate unsteady separated flows (periodic hill, low pressure turbine blade with and without inflow turbulence etc.)

- Diosady, Murman 2014, AIAA paper 2014-2784

- Diosady, Murman 2015, AIAA paper 2015-0294 


\section{Introduction}

Forcing Environmental Disturbances

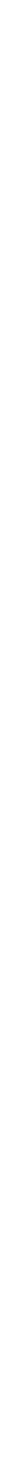




\section{Objective}

- Conduct scale-resolving simulations of HPT cascade to document effect of inflow turbulence on transition

- Arts et al. 1990 measured heat flux characteristic for a wide range of Reynolds number, Ma, inflow turbulence of an HPT blade

- RANS fails to predict transition characteristics, hence thermal load

- Previous DNS, LES, DESs also have difficulty on predicting transition and heat transfer characteristics

- To understand why numerical simulations fail to predict experimentally observed transition and heat transfer characteristics 


\section{Inflow-Outflow Boundary Condition Perfectly Matched Layer}

Instantaneous total pressure

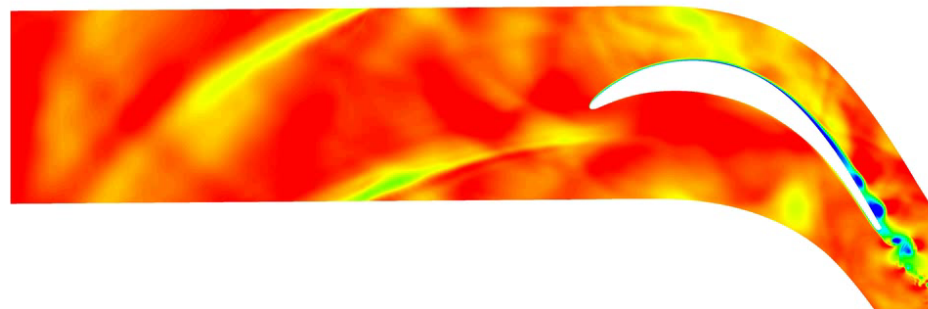

Without PML

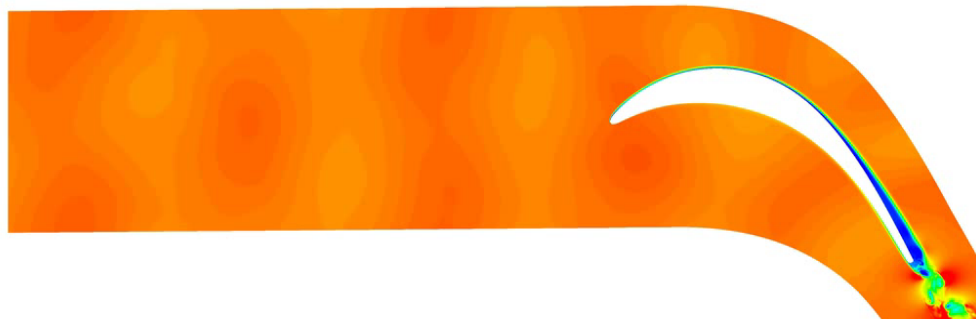

With PML

- Proper BC specification at artificial computational boundaries a major challenge especially for DNS or LES of unsteady turbomachinery flows

- Spurious acoustic reflections from boundaries can contaminate simulations

- Effect of reflections is severe for high-order low-dissipation schemes

- Authors successfully implemented the PML approach of Parrish and $\mathrm{Hu}$ in DG framework (presented at SciTech 2016) 
- HIT-based "linear forcing" method

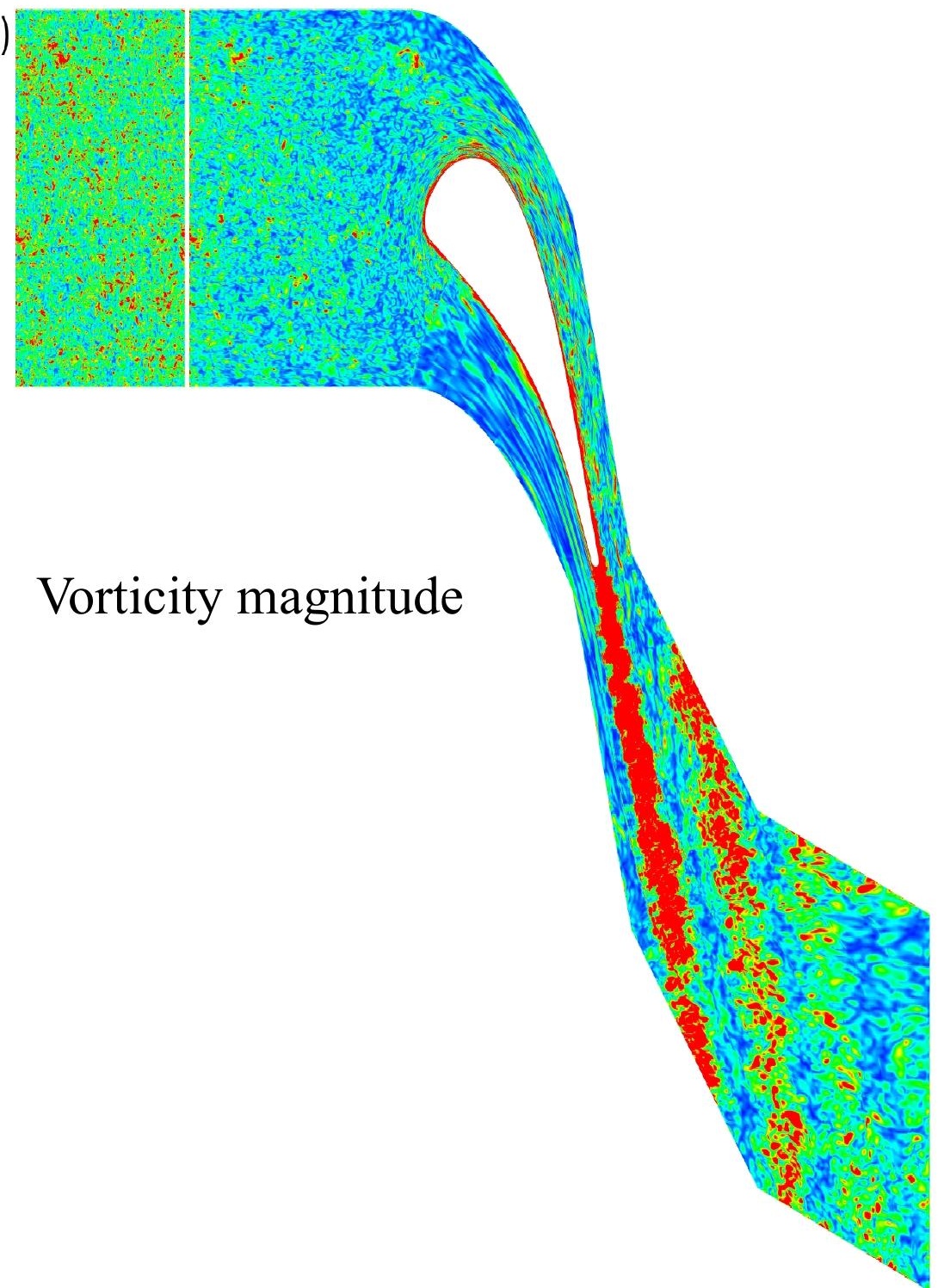

- Extension of linear forcing to anisotropic domains a challenge since all the wavenumbers are forced

- Multiple, stacked, individuallyforced cubes used to avoid largescale turbulent structures

- Generated turbulence is fed into the computational domain by solving a numerical Riemann problem

- Introduces recycling scale in the main computational domain

- Recycling scale is at least one eddyturnover time of HIT by stacking HIT cubes in streamwise direction 


\section{Problem Setup}

$4^{\text {th }}$ order

$8^{\text {th }}$ order

$16^{\text {th }}$ order
- $\operatorname{Re}=10^{6}$

- $\mathrm{Ma}_{\mathrm{e}, \mathrm{is}}=0.7$

- Experimental Tu 1\%,6\%

- Spanwise extent 20\%C

- Present simulations Tu 0\%,7\%, 20\%

- PMLs are applied at the inflow and outflow for $0 \% \mathrm{Tu}$

- Inflow turbulence length scale not reported in the experiment

- For present simulations we consider $4 \% \mathrm{C}$ as inflow turbulence length scale

- Results in $\operatorname{Re}_{\lambda}$ of 62 and 110 for $7 \%$ and $20 \% \mathrm{Tu}$

- Inflow turbulence is generated using linearforcing method

- For nonzero Tu, PML is applied at the outflow only

- Different mesh resolutions are used for different $\mathrm{Tu}$ 


\section{Flow visualization}

Vorticity magnitude in the xy plane Heat flux on the airfoil
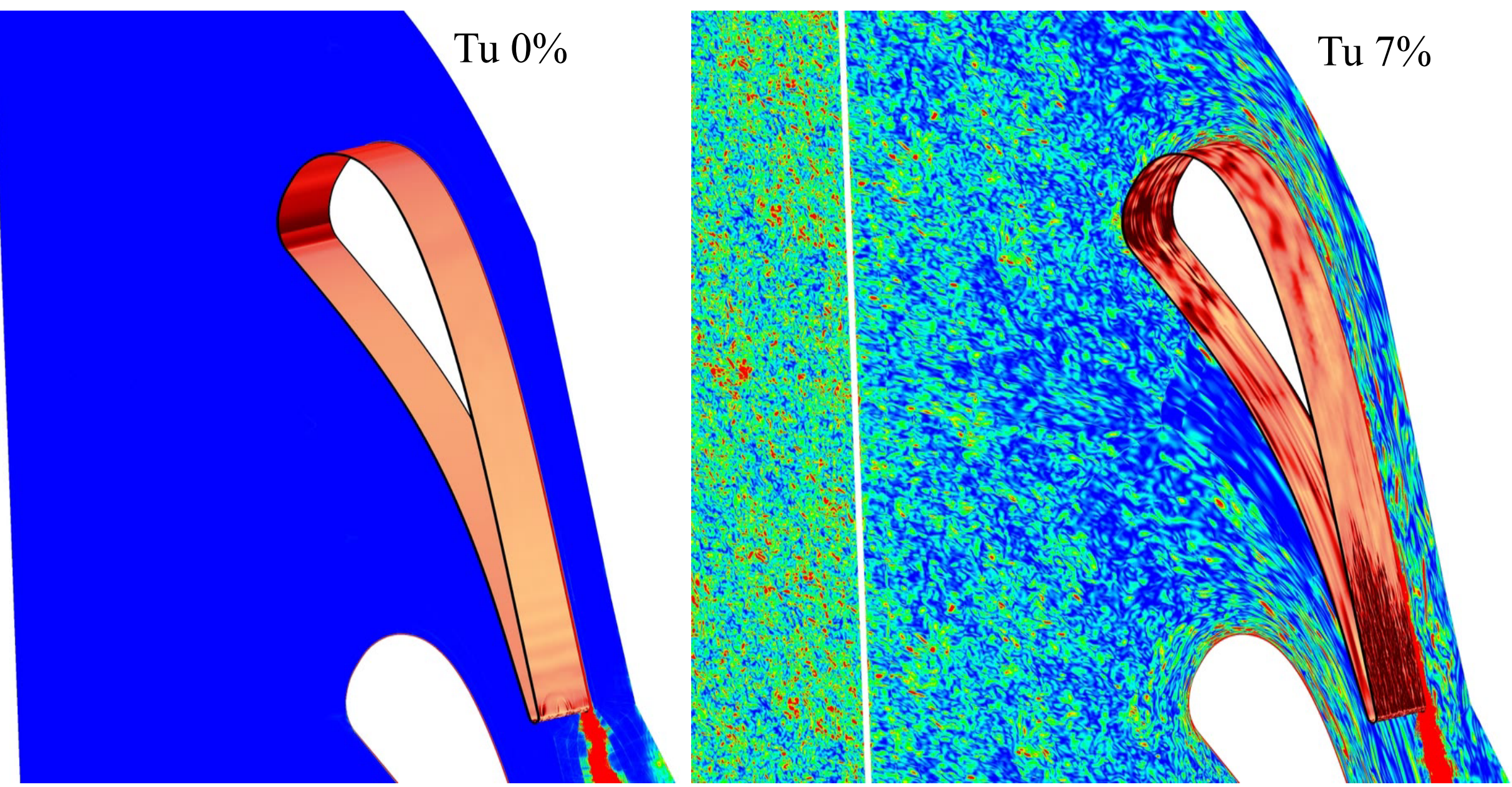

- High heat flux fore section of the airfoil

- Spanwise 2D TS waves, 3D turbulence close to the trailing edge for clean flow

- Streamwise Klebanoff modes, turbulent spots for $7 \% \mathrm{Tu}$

- Breakdown to turbulence is highly intermittent 


\section{Comparison with the experiment and RANS}




\section{Mean Heat flux}

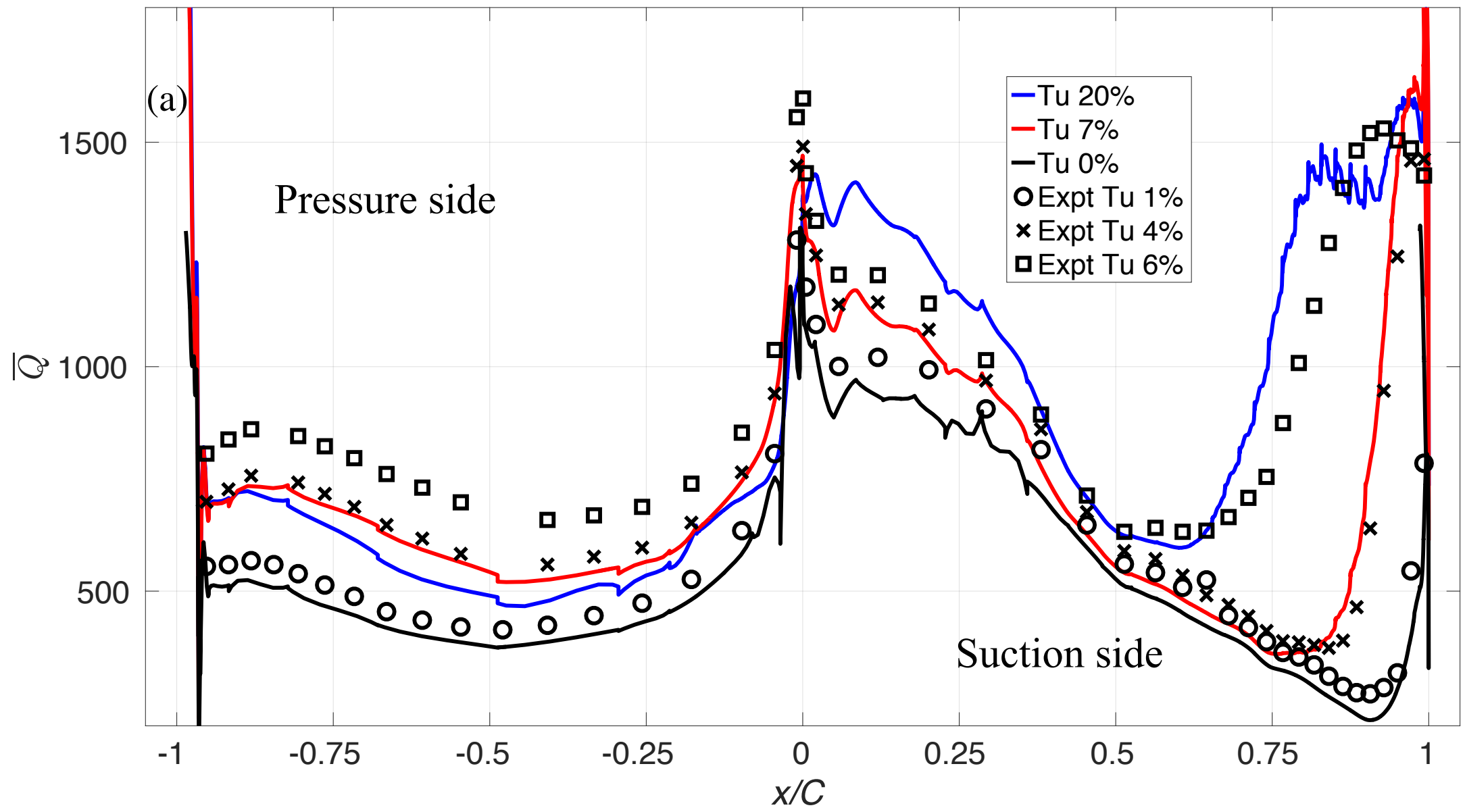

- Heat flux peaks at the leading edge, and turbulent flow region of the suction side

- $0 \% \mathrm{Tu}$ agrees well with the experiment

- $7 \% \mathrm{Tu}\left(\mathrm{Re}_{\lambda} 62\right)$ agrees with the experimental $4 \% \mathrm{Tu}$

- $20 \% \mathrm{Tu}\left(\operatorname{Re}_{\lambda} 110\right)$ agrees with the experimental $6 \% \mathrm{Tu}$ 


\section{Mean Skin friction}

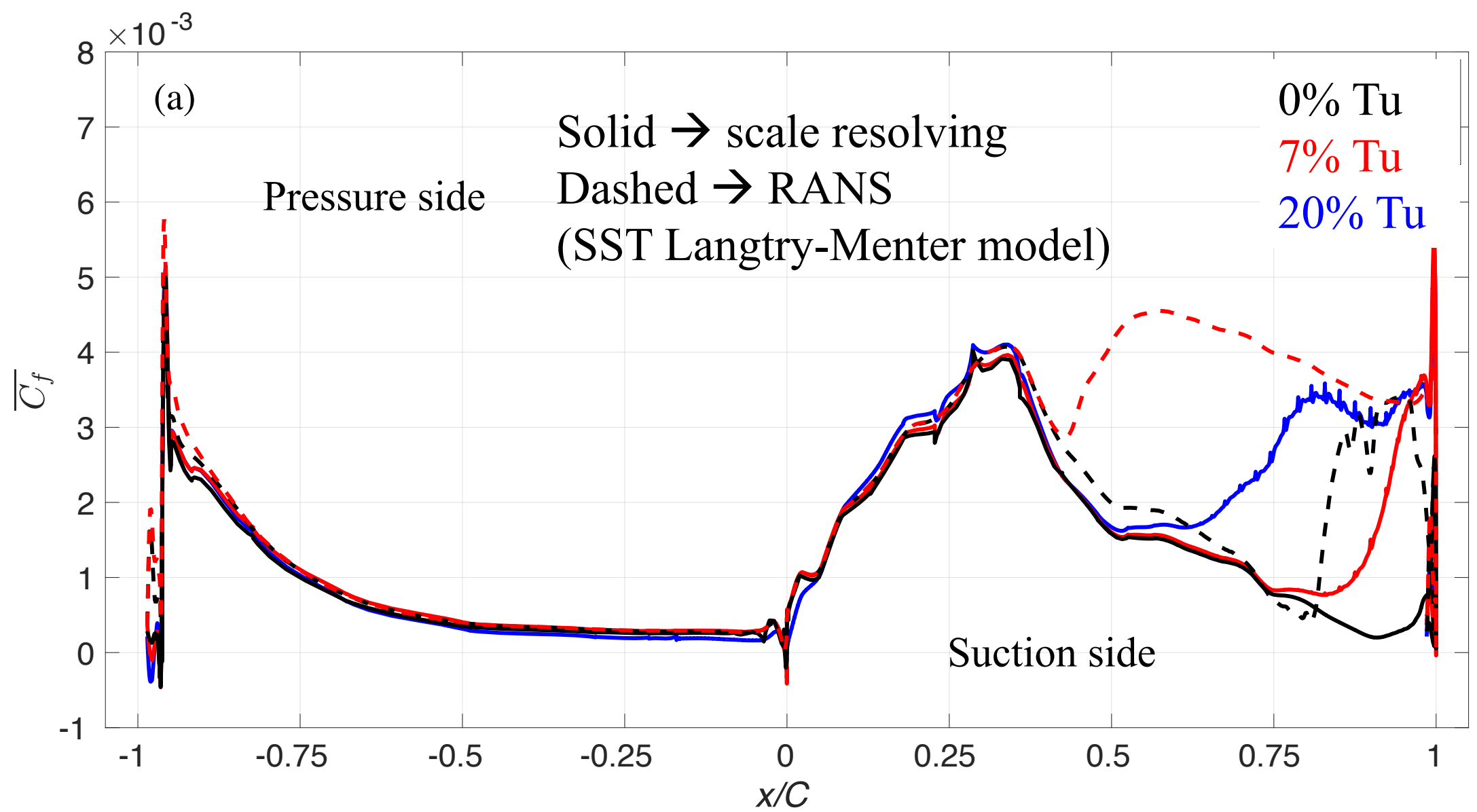

- Unlike heat flux, friction coefficient peaks at the suction peak, and at the turbulent region

- RANS predicts transition location further upstream compared to experimental observation, even for $0 \% \mathrm{Tu}$ 


\section{Heat flux unsteadiness}

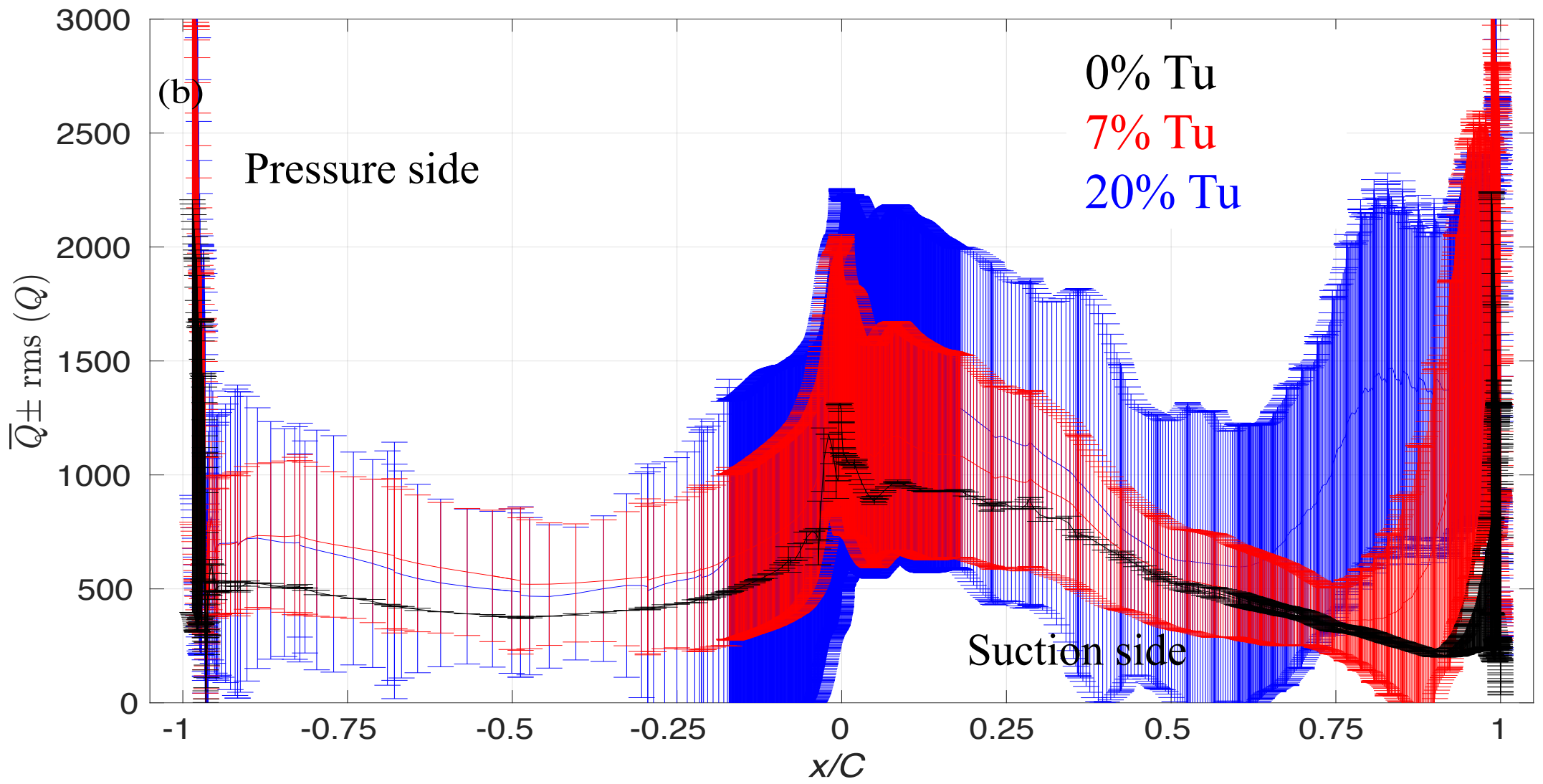

- For $0 \% \mathrm{Tu}$, heat flux unsteadiness is negligible except near the trailing edge, where transition occurs

- For high Tu, high heat flux unsteadiness is present even before the transition occurs, due to Klebanoff modes

- Instantaneous heat flux is much greater than the mean heat flux 
Suction side boundary layer 


\section{Turbulent kinetic energy}

(a) 0.0

$\mathrm{Tu} 0 \%$
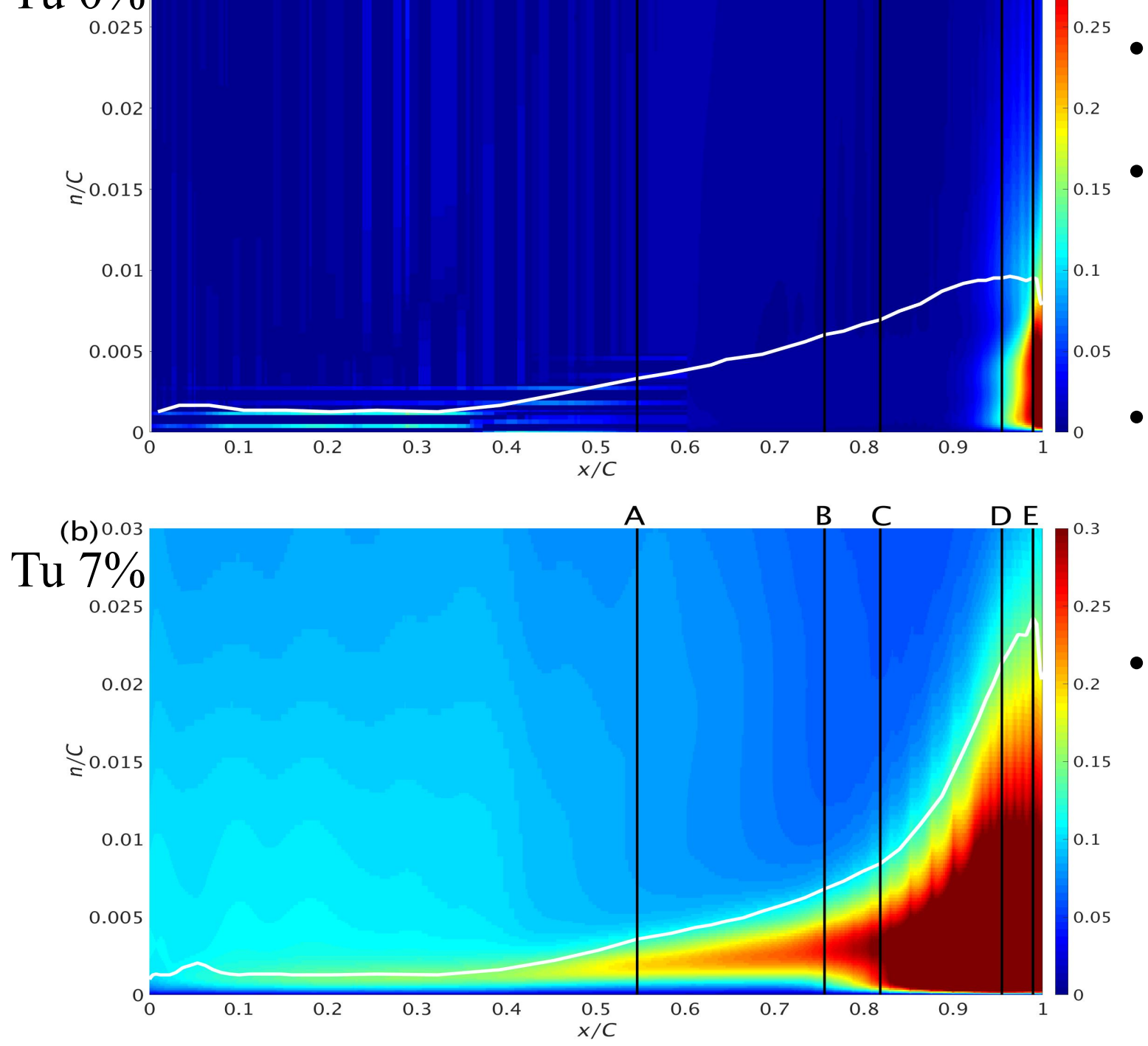

- For $0 \% \mathrm{Tu}$, tke is high close to the trailing edge

- For $7 \% \mathrm{Tu}$, high tke is present at the boundary layer edge at the fore section of airfoil $\rightarrow$ Klebanoff mode

- This high tke region penetrates towards the wall $\rightarrow$ 'top-down' mechanism of bypass transition

- Boundary layer thickness grows rapidly as boundary layer transitions 


\section{Shape factor}

\section{Tu 0\%}
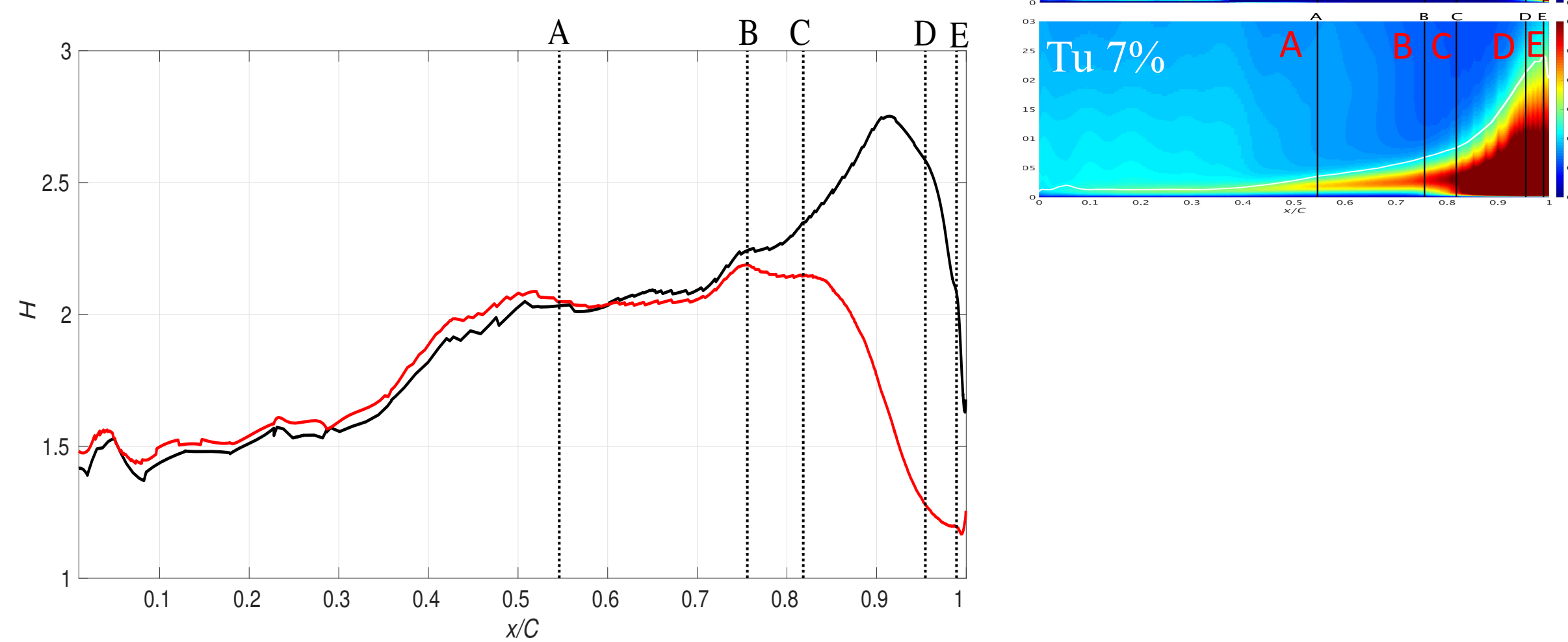

- Shape factor is much less than Blasius value before suction peak, where flow accelerates

- After suction peak it starts to increase

- For clean inflow it attains Blasius profile value before transition occurs

- As flow transition occurs, it drops to the turbulent boundary layer value 


\section{Reynolds stress profile}

\section{$\mathrm{Tu} 0 \%$}

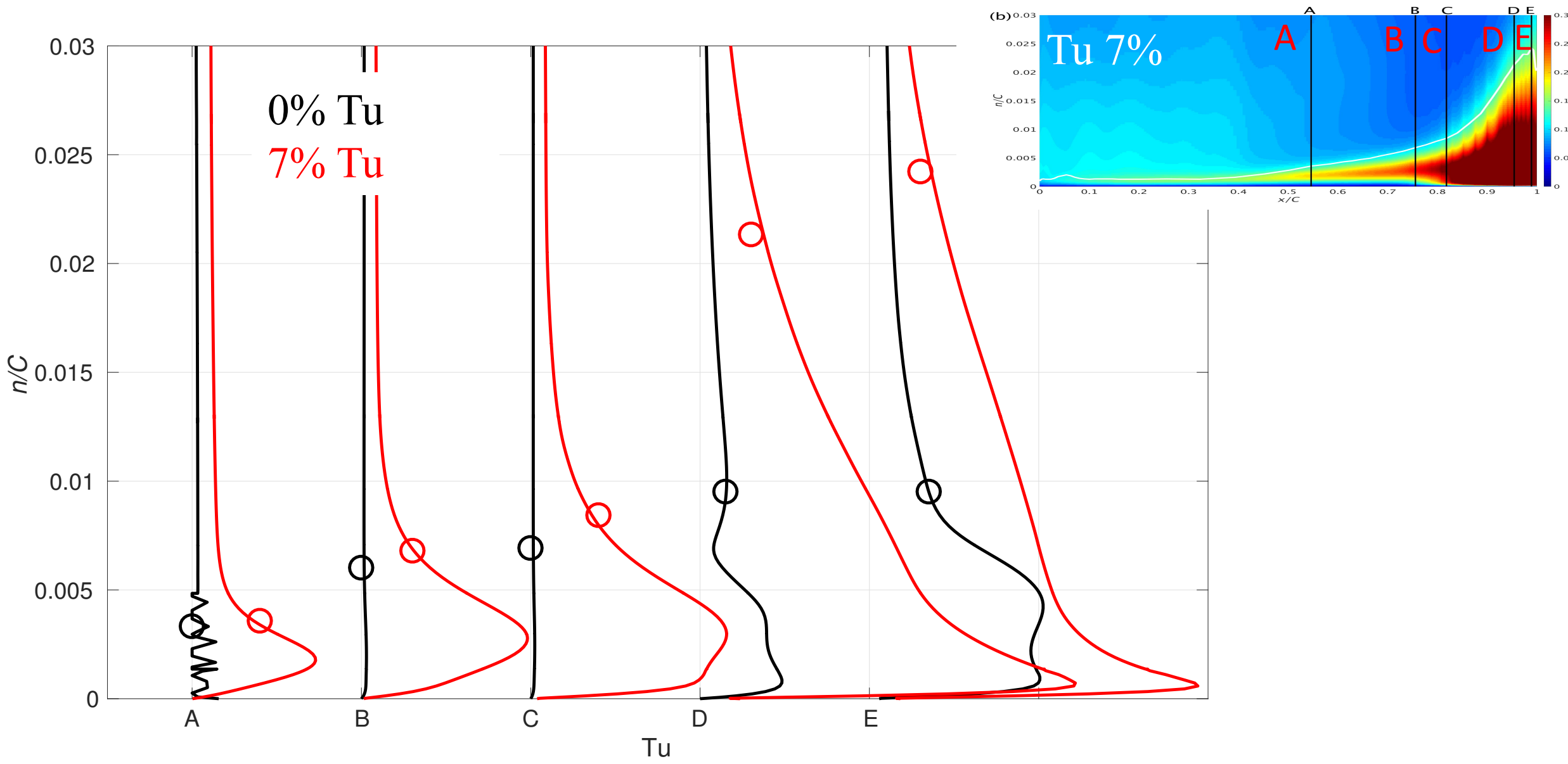

- $0 \% \mathrm{Tu}$ tangential profiles shows signature of most unstable mode for TS waves (primary peak near the wall, secondary peak near the boundary layer edge)

- $7 \%$ Tu has much higher values. Before transition it peaks close to the the boundary layer edge, after transition it peaks close to the wall 


\section{Lumley triangle}
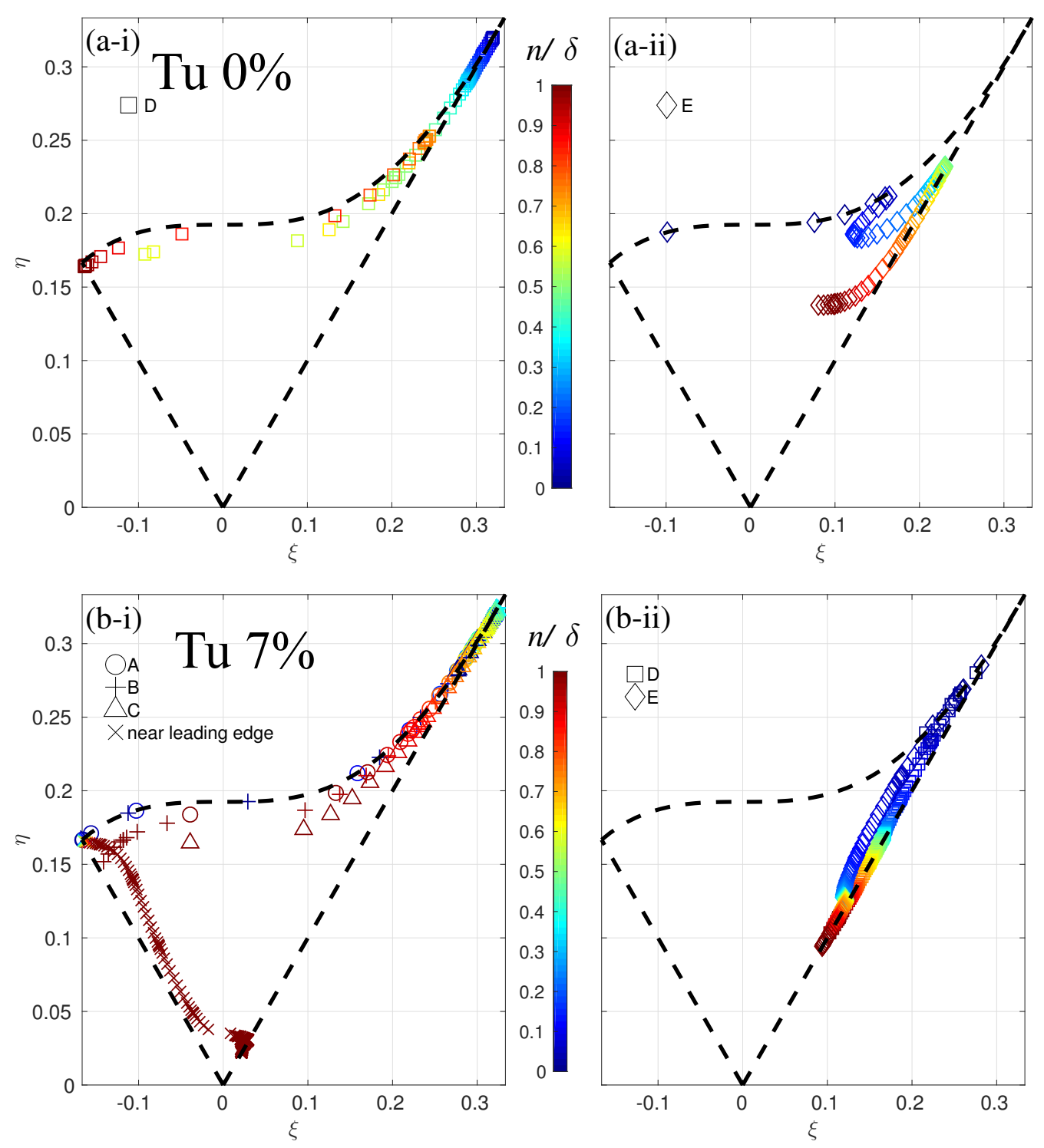

\section{Tu $0 \%$}

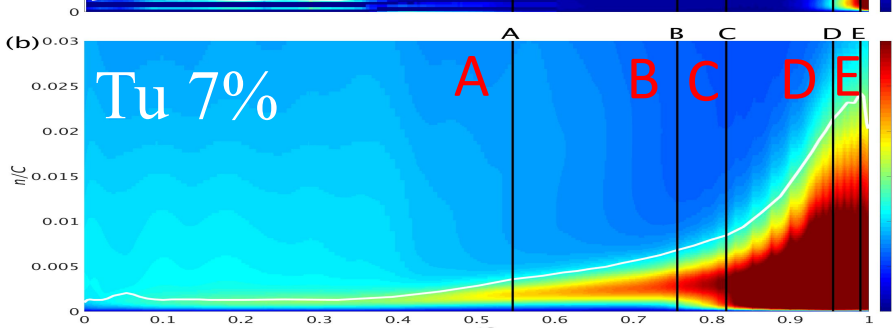

- Reynolds stress characteristics are different during the transition process for natural and bypass transition

- Inflow turbulence has isotropic structures that undergo strong stretching due to flow acceleration

- For 7\% Tu, when the boundary layer becomes fully turbulent, the characteristics matches that of turbulent channel flow 


\section{TKE budget: $0 \% \mathrm{Tu}$}
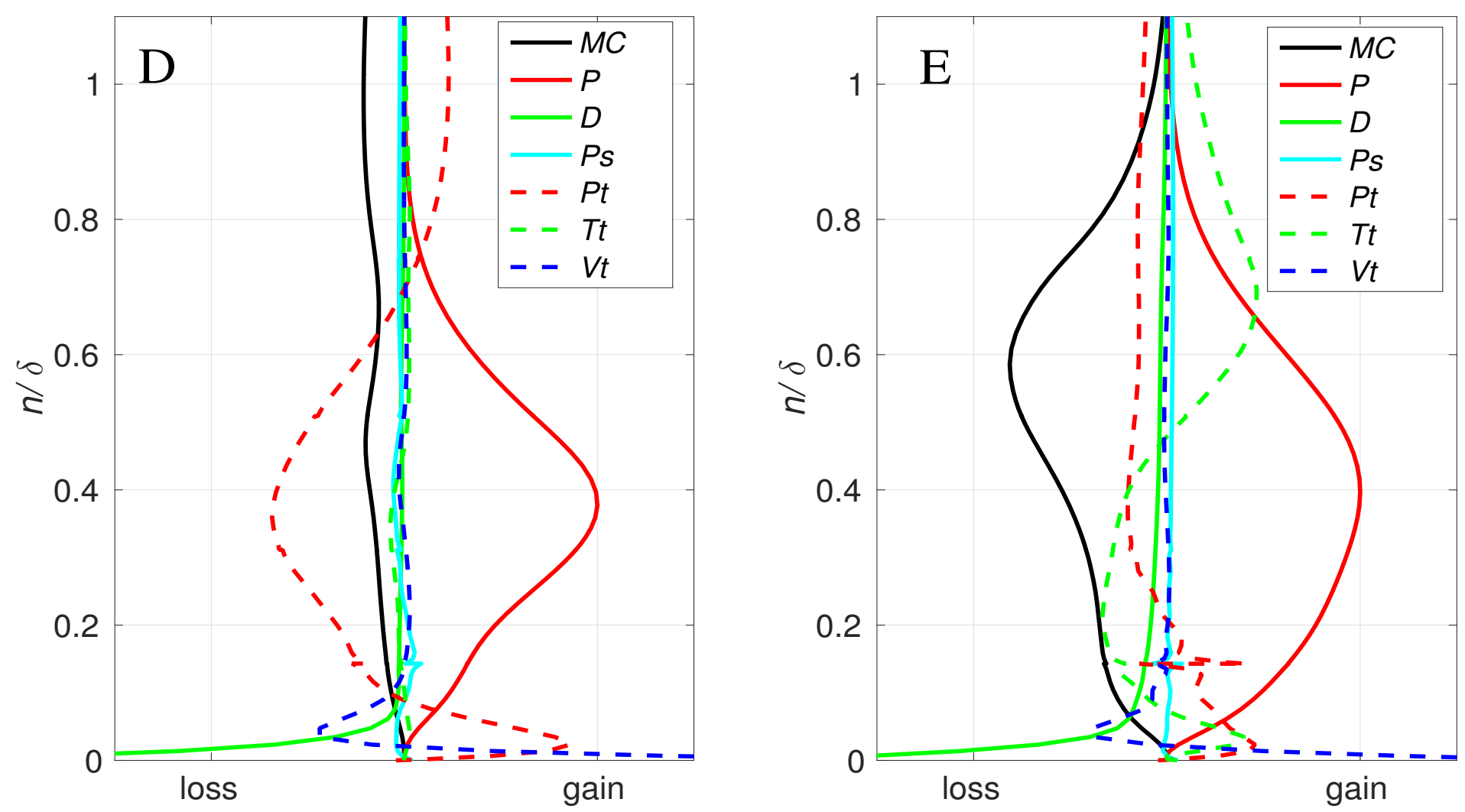

- Pressure transport plays significant role for initial phase of TS wave propagation

- Mean convection balances production and turbulent transport during the transition for majority of the boundary layer 


\section{Summary}

- Studied the natural and bypass transition for high pressure turbine airfoil

- $0 \%, 7 \%\left(\operatorname{Re}_{\lambda}=62\right), 20 \%\left(\operatorname{Re}_{\lambda}=110\right)$ Tu results agree well with the experimental $1 \%, 4 \%$ and $6 \% \mathrm{Tu}$

- Turbulent structures result in unsteady heat flux, comparable to the mean at the airfoil

- Mean heat flux and skin friction distributions are different near the leading edge

- Turbulent characteristics are different for natural and bypass transition 


\section{Future Work}

- Looking for better documented experimental result

- Wall modeled LES with transition models

- Efficient generation of inflow turbulence

- Adjoint driven mesh adaptation

\section{Acknowledgment}

- NASA Advanced Air Transport Technology Project, Advanced Air Vehicles Program

- NASA Advanced Supercomputing (NAS) facility at NASA Ames Research Center
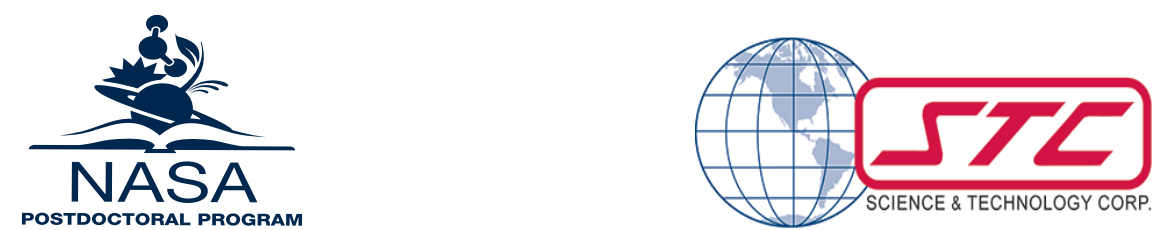


\section{Thank You}

$0 \% \mathrm{Tu}$

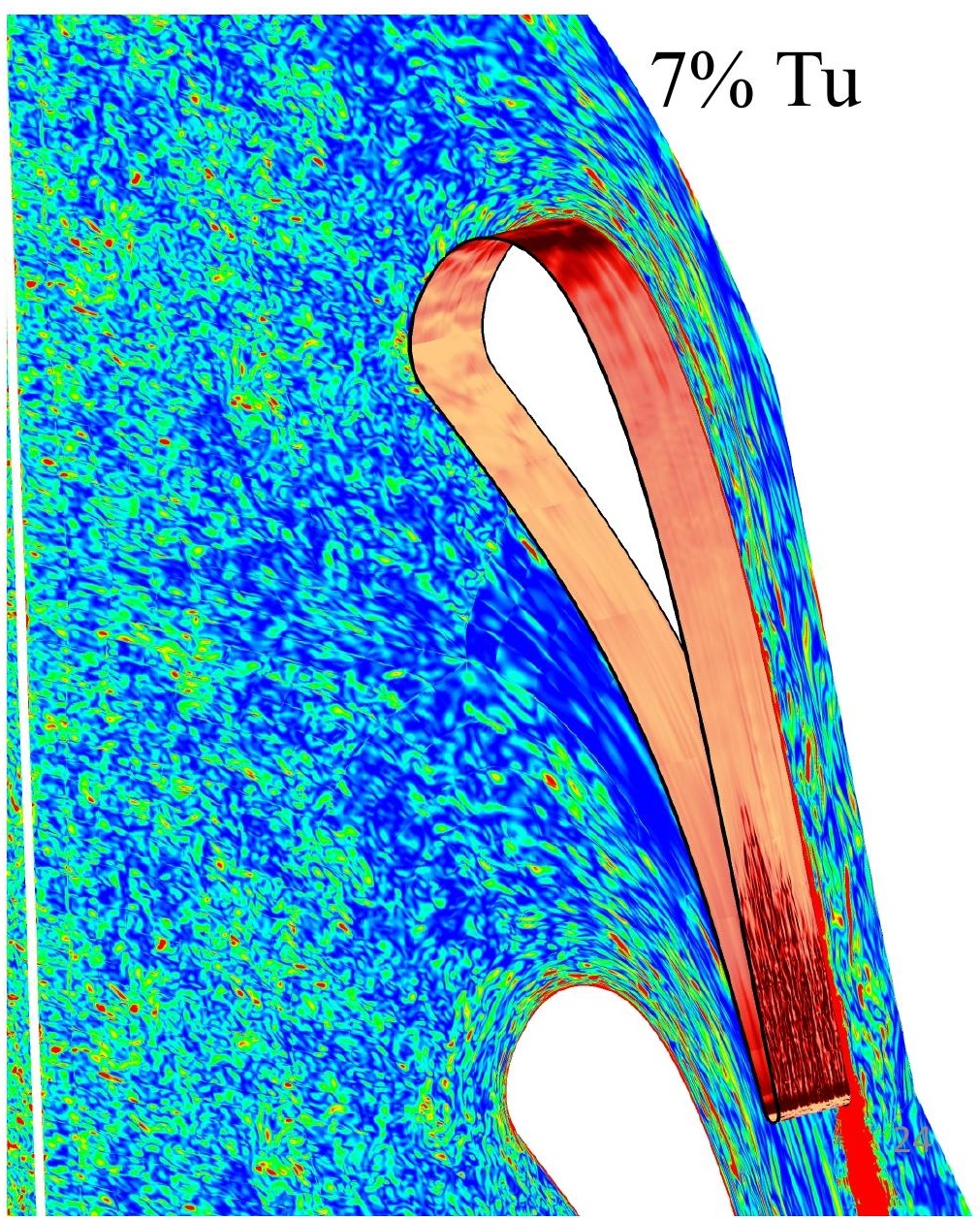

